\title{
Estimation of Data-Dependent Jitter Using Single Pulse Analysis Method in High-Speed Differential Signaling
}

\author{
Eakhwan Song, Junho Lee, Jingook Kim, Dong Gun Kam, Chunghyun Ryu and Joungho Kim \\ Terahertz Interconnection and Package Laboratory \\ Dept. of EECS, KAIST,371-1 Gusung,Yusung,Daejeon 305-701, Korea \\ Phone) +82-42-879-9868, Fax) +82-42-869-8058, E-mail) sehnx@eeinfo.kaist.ac.kr;teralab(a)ee.kaist.ac.kr
}

\begin{abstract}
Timing margin in high-speed data channel becomes tighter as bit rate goes higher. Timing jitter pares down the timing margin and makes it difficult to set timing budget for the receiver to distinguish digital binaries. Especially, the timing jitter that is correlated with the bit sequence in a data stream is called data-dependent jitter (DDJ). DDJ rises up with higher bit rate in dispersive lossy channel, therefore the issues about DDJ are rising up in recent high-speed serial interface design. In this work, a simple analysis method for the estimation of DDJ in high-speed differential signaling using single bit pulse response is proposed. A frequency dependent model of a transmission line is considered to characterize lossy properties of the differential channel, and the single bit pulse response is obtained with the model. The estimated DDJ in the differential signaling is experimentally verified with jitter measurements.
\end{abstract}

\section{Introduction}

Almost of the high-speed serial interfaces provide the margin of timing jitter in technical specifications as a standard of compliance test. The vendors producing high-speed interconnects have to pass the compliance test with guaranteeing the specification of timing jitter to put the products into the market. Especially, the timing jitter which is correlated with bit-sequence in data stream is called data-dependent jitter (DDJ) [1], and this metric becomes more important issue as data-rate goes up. When designing high-speed channel, it would be so fascinating that the designers could estimate datadependent jitter without production and real measurements for the compliance test. Therefore, they can be more competitive saving the cost for production and time to market.

For designing high-speed channel with efficiency, many trials to predict data-dependent jitter have been continued. The most common process among them is to utilize commercial tool for simulations. However, this way takes much time for fine timing resolution and does not give the choice of the models for simulation except the ones which are provided from the tool from itself. So the analytic approach is needed for the estimation of data-dependent jitter. There have been proposed some analytic estimating method of data-dependent jitter, however, which have been common problem that the bandwidth of the channel should be known in advance [2][3]. Thus bandwidth limiting filter is used for fixing the bandwidth of the channel for mathematical models. To avoid the ideal assumption, we physically characterize the channel based on the transmission line theory and then proposed single pulse analysis method for the estimation of data-dependent jitter. The single pulse analysis method is based on the worst case analysis of high-speed data transmission, so the calculation time is remarkably reduced to that of the previous analytic methods with maintaining the flexibility for characterizing the model of the channel by numerical calculation instead of the analytic solutions. In this paper, the method of giving simple estimation of the data-dependent jitter which could be used as a pre-compliance test is proposed in designing level.

\section{Inter-symbol Interference}

With any practical channel, the inevitable lowpass filtering effect causes a spreading of individual data symbols passing through a channel. And this smeared symbol can corrupt another symbol traveling on the same channel at a later time, this phenomena is called as inter-symbol interference (ISI).

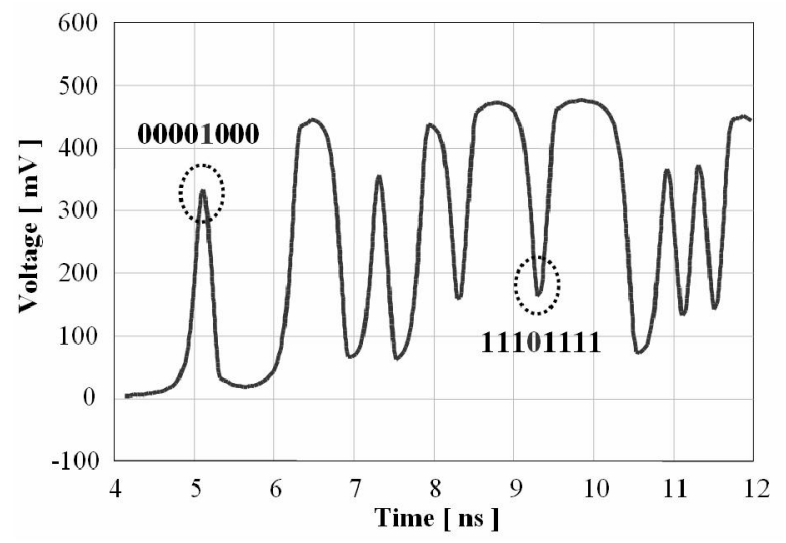

Figure 1 Distorted waveform of a data due to ISI 
This inter-symbol interference can significantly degrade the quality of a data and makes receiver hard to differentiate a current symbol from the diffused energy of the adjacent symbols. Figure 5 shows the random data with bit rate of $5 \mathrm{~Gb} / \mathrm{s}$ after flying $50 \mathrm{~cm} \mathrm{u}$-strip line. Because of the dispersive lossy characteristic of the channel, the effect of inter-symbol interference is well shown in the figure. And it is easily found that the response of a single bit pulse after consecutive 0 's or 1's is the response of the worst case.

\section{Single Pulse Analysis Method}

A clock signal has no data-dependent jitter because it has actually no data-pattern but periodic. So the clock signal can be used as a reference which determines the ideal timing position, and the datadependent jitter can be obtained by comparing the single pulse response with the clock.

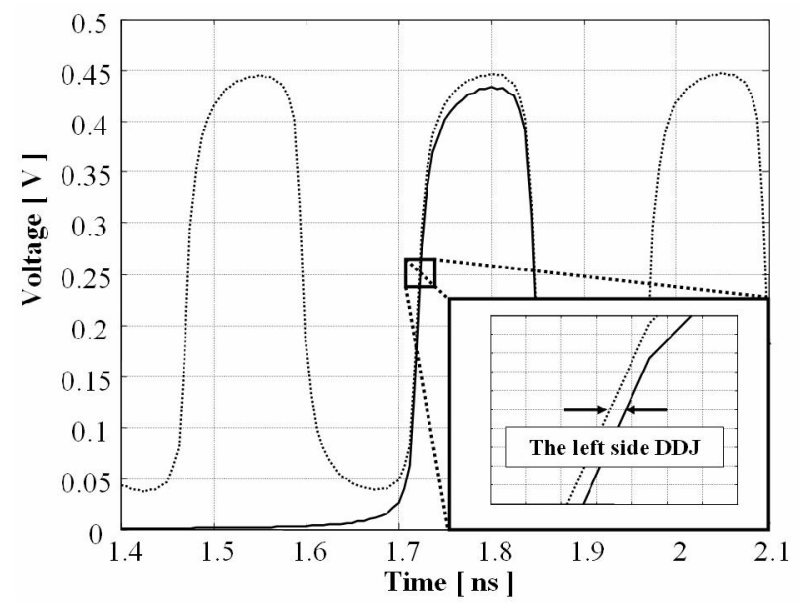

Figure 2 Comparison of a clock signal and the single pulse response

In Figure 2, the output of the single pulse rises later than that of a periodic clock signal and falls earlier in the same manner. These discrepancies of the reference-crossing time between the single pulse response and periodic clock pattern represent the both side of data-dependent jitter in the worst case and the total data-dependent jitter becomes the sum of them (1). And it is simply found that the difference between the bit period and the timing width of the single pulse response also implies the total data-dependent jitter (2). These relations are shown in Figure 3.

$$
\begin{aligned}
t_{D D J} & =t_{D D J_{-} \text {left }}+t_{D D J_{-} \text {right }} \ldots \ldots \ldots . \\
& =t_{\text {bit }}-t_{\text {eye width }} \quad \ldots \ldots \ldots \ldots \ldots \ldots
\end{aligned}
$$

$$
\text { where, }\left\{\begin{array}{l}
t_{D D J}: \text { the total } D D J \\
t_{D D J_{\text {_left }}}: \text { the left side DDJ } \\
t_{D D J_{\text {_right }}}: \text { the right side DDJ }
\end{array}\right.
$$

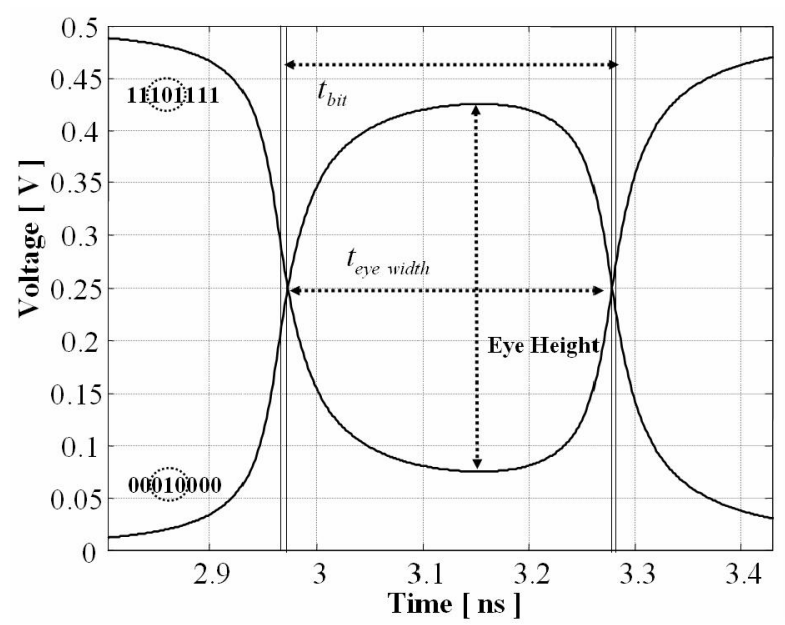

Figure 3 Single pulse response at bit rate of $3.2 \mathrm{~Gb} / \mathrm{s}$ after flying through a $50 \mathrm{~cm} \mathrm{u-strip} \mathrm{line}$

It is hard to get the single pulse response as closed-form equation because the frequencydependent characteristic of the channel is so complex. One method to dealing with this problem is to use fast-fourier transformation (FFT) and the inverse of it (iFFT) and then numerically performs the conversion of one domain into the other. This has advantages for flexibility for choosing the model of the channel. If we got more suitable frequencydependent model of the channel, we could apply the model easily just by substituting it with old one.

First, we model the single pulse in time domain and it is fast-fourier transformed into a stimulus in frequency domain, $X(\mathrm{f})$. And this input stimulus is multiplied with the transfer function of the channel to construct output response Y(f). Then the final single pulse response in time-domain can be derived by inverse fast-fourier transformation of it. This is the one of traditional procedures which performs transient simulation using frequency domain data obtained by measurement or frequency-dependent modeling of a channel. The accuracy of the output response depends on the perfection of the models of single pulse and transfer function and the number of the calculation when fast-fourier transformation is carried out [Figure 3].

\section{Modeling of a Single Bit Pulse}

We modeled single pulse with empirical relation between the rise time of a input signal and the bandwidth of the interconnection. If we are aware of all bandwidth of the all parts of the system, we can 
obtain the inherent rise time respectively with this relation (3) [4]. For example, our measurement setup is composed of pulse pattern generator which has rise time less than $30 \mathrm{ps}$, cables for assembly guaranteeing the bandwidth of $46 \mathrm{GHz}$ and the sampling oscilloscope with the sampling rate of $20 \mathrm{GHz}$.

In this step, the input single pulse should be modeled considering all parts of the system except the channel which would be modeled in next step. Suppose the rise time of the worst case from pulse pattern generator (PPG) is 30ps, and the rise time from the other parts can be derived simply (4). Total inherent rise time can be got by root-sum-square of the rise time of from each part except the channel (5). In case of this setup, the total inherent rise time is calculated as 36.90 ps.'

$$
\begin{aligned}
& R T(\text { rise time })=\frac{0.35}{B W(\text { bandwidth })} \quad \ldots . . . . . .(3) \\
& R T_{1}=30 \mathrm{ps}(\text { from } P P G) \\
& R T_{2}=0.35 / 46 \mathrm{GHz}=7.61 \mathrm{ps}(\text { from cables }) \\
& R T_{3}=0.35 / 20 \mathrm{GHz}=15.17 \mathrm{ps}(\text { from oscilloscope })
\end{aligned}
$$

$$
R T=\sqrt{R T_{1}^{2}+R T_{2}^{2}+R T_{3}^{2}}=36.90 p s
$$

After getting the rise time, the single pulse can be modeled by composing two $\mathrm{RC}$ step responses which have opposite polarity and delay of a bit period (6) [Figure 4]. And the total inherent rise time determines the time constant. The $\mathrm{RC}$ step responses are enough to express the input single pulse because there is an assumption that no dispersion appears in all parts of the system except the channel which would be modeled in next step. Actually, the cables and connectors used in this setup $3.5 \mathrm{~mm}$ type, that means the dielectric material is air which has no dispersive characteristics; dielectric constant of the air almost has no variation over wide frequency range. This single pulse is regarded as an input stimulus holding all characteristics of the system except the channel [Figure 5].

$$
V_{\text {out }}(t)=A\left(1-e^{-t / \tau}\right)
$$

where, $\left\{\begin{array}{l}V_{\text {out }}: \text { Output Voltage } \\ A: \text { Amplitude } \\ \tau: \text { Time Cons } \tan t\end{array}\right.$
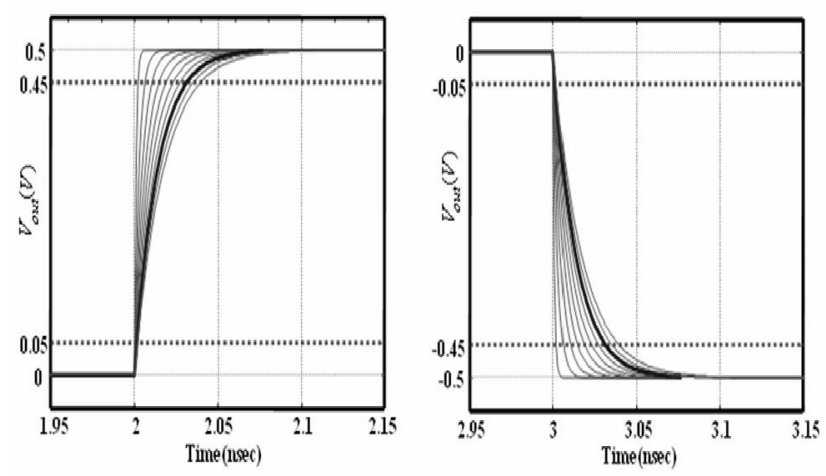

Figure 4 RC step responses composing single pulse

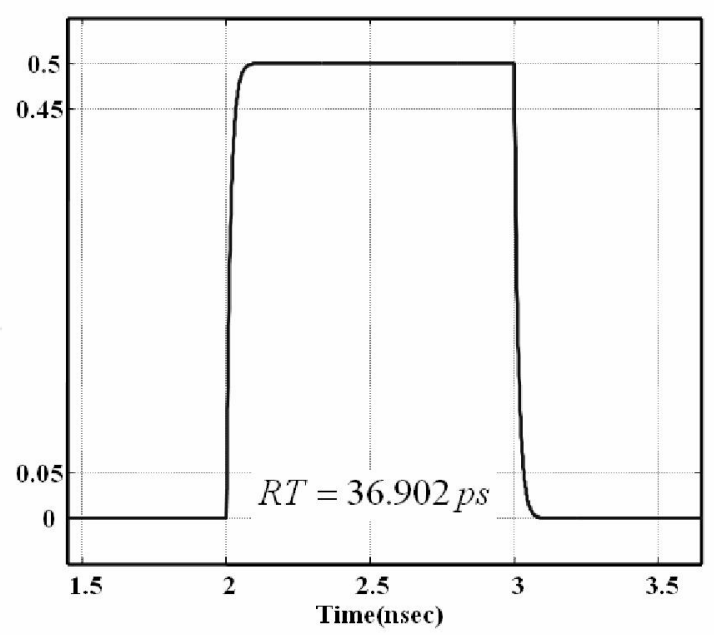

Figure 5 Single pulse as an input stimulus

\section{Modeling of High-speed Differential u-strip Line}

Especially copper based interconnection without low-k substrate material suffers from timing jitter at multi-gigabit wideband signals through frequencydependent loss. Both skin-effect and dielectric loss are the representative causes to degrade the quality of the signals which commonly used in current highspeed digital system. These losses make signal distorted, and this distortion is shown up as intersymbol interference which is the source of datadependent jitter. And through the process of converting the frequency domain into the time domain, some sources of error can be generated; non-causality, bandwidth limit and others. So wellconsidered frequency-dependent model of the channel is needed and frequency-dependent loss is also important. The channel used in this work is simple differential u-strip line. In point of views of single-ended line, frequency-dependent model of the line is reliably characterized in [5]. For expanding the model to differential line, some additional parameters are to be considered.

Typically, differential lines are quite close to each other, enough to make interactions between them. And these interactions induce the 
electromagnetic field distribution between the differential pair. Therefore, propagation of the signal through the differential pair cannot be described with a single capacitance and inductance but considering the interaction. And if there are any discontinuities in differential pair, it cannot be considered as complete differential mode with generating common mode noise. It is too difficult to predict and model the differential impedance discontinuities, so those are ignored in this work. Actually, the channel used to verify the method is simple u-strip line, the assumption that no common mode noises are generated is reasonable.

Figure 6 shows the electric field distributions on differential transmission in the even mode and the odd mode. $C_{p}$ and $C_{f}$ are line capacitance with parallel plate and fringing capacitance which are common with those of even mode signaling, and $C_{g d}$ and $C_{g a}$ are the gap capacitance through the dielectric materials and the air which represent the interaction between the differential pair [6].

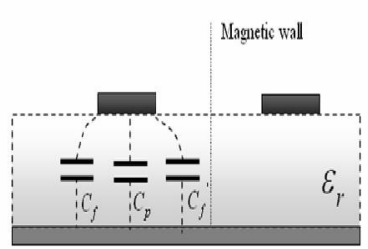

(a)

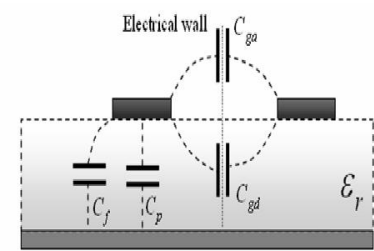

(b)
Figure 6 (a) Even mode and (b) odd mode capacitances on differential signaling

Those capacitances are parallel each other, so the total even mode and odd mode capacitance are derived from the sum of them respectively (7).

$$
\begin{aligned}
& C_{e}=C_{p}+C_{f}+C_{f} \\
& C_{o}=C_{p}+C_{f}+C_{g d}+C_{g d}
\end{aligned}
$$

With these even mode and odd mode capacitances, the self and mutual capacitances can be obtained by (8) [7]. And the self and mutual inductances are also easily calculated consequently by the relation of speed of light with them (9). Finally, the propagation constant in odd mode is represented as (10), and it can be applied to single pulse analysis method by regarding it as propagation constant in differential signaling.

$$
\begin{aligned}
& C_{\text {self }}=\left(\frac{C_{o}+C_{e}}{2}\right), \quad C_{m}=\left(\frac{C_{o}-C_{e}}{2}\right)(F / m) \\
& {\left[\begin{array}{ll}
L_{\text {self }} & L_{m} \\
L_{m} & L_{\text {self }}
\end{array}\right]=\mu_{0} \varepsilon_{0}\left[\begin{array}{ll}
C_{\text {self_a }} & C_{m_{-} a} \\
C_{m_{-} a} & C_{\text {self_a }}
\end{array}\right]^{-1}(\mathrm{H} / \mathrm{m})} \\
& \gamma_{\text {odd }}=\sqrt{\left(j \omega\left(L_{\text {self }}-L_{m}\right)+r\right)\left(j \omega\left(C_{\text {self }}+C_{m}\right)\right)}
\end{aligned}
$$

Figure 8 compares SDD21 between the circuital differential channel model and the results of the 3D field simulator (CST MWS). It shows good correlation up to $10 \mathrm{GHz}$, so there is no issues to utilize the model of differential channel validating with the data rate up to $3.2 \mathrm{~Gb} / \mathrm{s}$ in the same manner with the case of single-ended channel in chapter 2. The validating setup is shown in Figure 7.

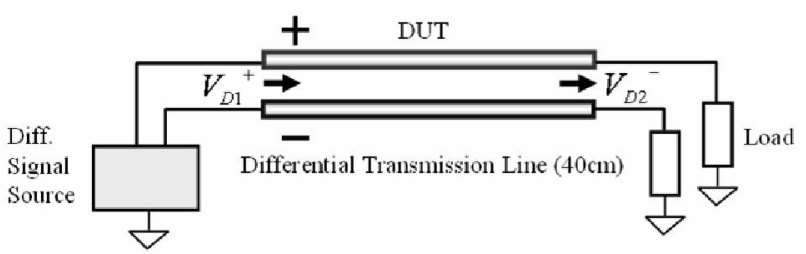

Figure 7 Setup for validating the differential transfer function

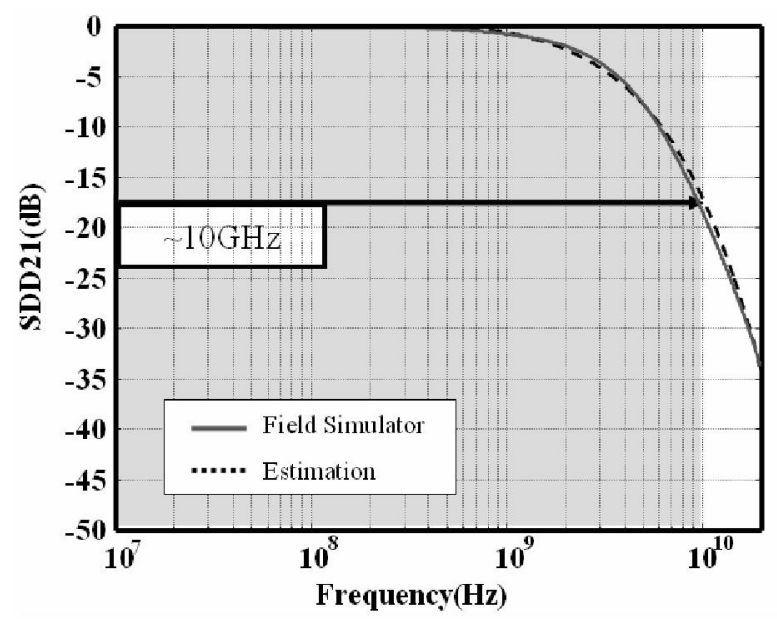

Figure 8 Comparison of the differential transfer function with the result of $3 \mathrm{D}$ field simulator

\section{Experimental Verification of the Method with Jitter Measurement}

We have performed the measurement on $40 \mathrm{~cm}$ FR4 based u-strip line varying bit rate of the data. The input signal was a $2^{\wedge} 7-1$ pseudo random bit sequence (PRBS) which has $500 \mathrm{mV}$ swing from 
pulse pattern generator (PPG). The output signal after flying through differential u-strip line was observed with Tektronix TDS8000B sampling oscilloscope equipped $20 \mathrm{GHz}$ sampling module. To avoid the parasitic effects of connectors, microprobes which have a cut-off frequency of $40 \mathrm{GHz}$ were used. The measurement setup is shown in Figure 9.

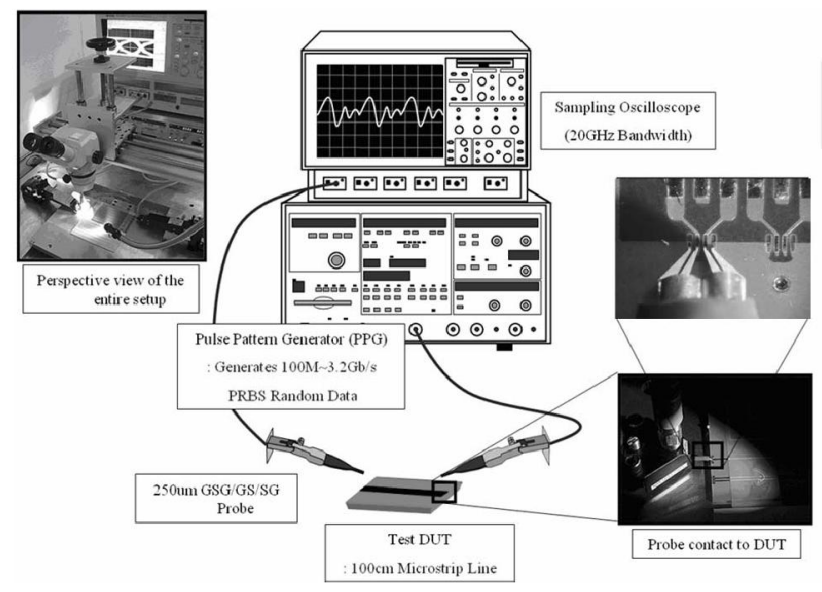

Figure 9 The entire measurement setup for experimental verification

When measuring jitter, the oscilloscope used in the measurement provides total jitter. That means the amount of the jitter measured from it includes that of the random jitter. With single pulse analysis method, only the data-dependent jitter can be estimated. Therefore, the data-dependent jitter should be extracted from the measured total jitter by deembedding random jitter. To get the data-dependent jitter from measurements, two kinds of measurements of each case were executed [Figure 10]. One is for the total jitter from the eye-diagram with random data, and the other is for the random jitter from single bit pulse pattern. No datadependent jitter appears in the latter, because there is only one pattern; single bit pulse pattern. Consequently, the data-dependent jitter can be obtained by subtracting the random jitter from the total jitter.
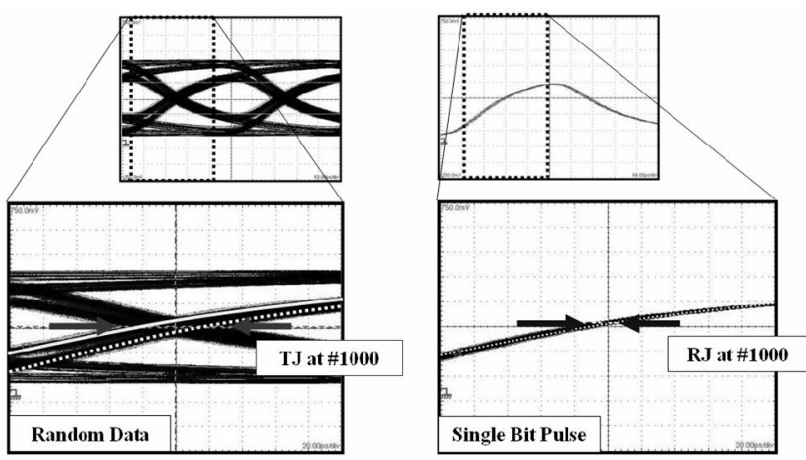

Figure 10 Data-dependent jitter extraction with deembedding random

Figure 11 shows the results of the data-dependent jitter and eye-height measurement along with the estimation using single pulse analysis in differential signaling. It can be found that there is good correlation between them, therefore, we can consider the single pulse analysis method is quite reliable with differential signaling.

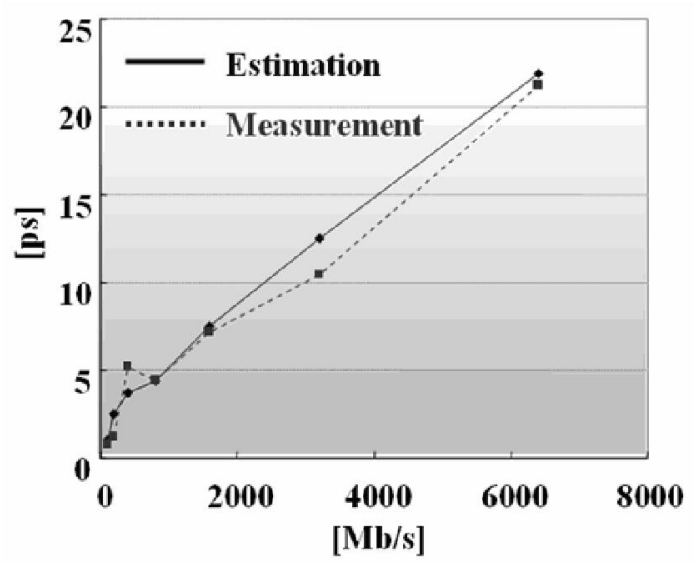

(a)

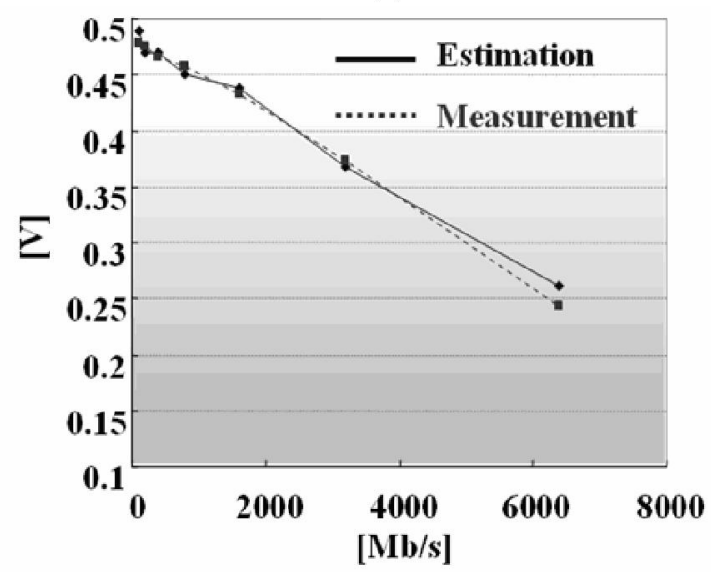

(b)

Figure 11 Comparison of the estimation of (a) datadependent jitter and (b) eye-height with the measurement with the bit rate scanned from $100 \mathrm{Mb} / \mathrm{s}$ to $6.4 \mathrm{~Gb} / \mathrm{s}$ in differential signaling 
If we are aware of all data pattern with single pulse response, the entire waveform can be obtained by convolution of them. Additionally, eye-diagram also can be constructed through superimposing the waveforms split from the total waveform with fixed timing window. And data-dependent jitter and eyeheight can be estimated with the eye-diagram because the worst case pattern is certainly shown in the eye-diagram. Actually, there is no need to construct eye-diagram to estimate data-dependent jitter but for the visual comparison of the estimations with the measurements. Figure 12 shows estimated and measured eye-diagram of $6.4 \mathrm{~Gb} / \mathrm{s}$ random data transmitted through $40 \mathrm{~cm}$ differential u-strip line. We could also find visual correlation of them. The bold solid line implies single pulse response which data-dependent jitter can be directly derived from.

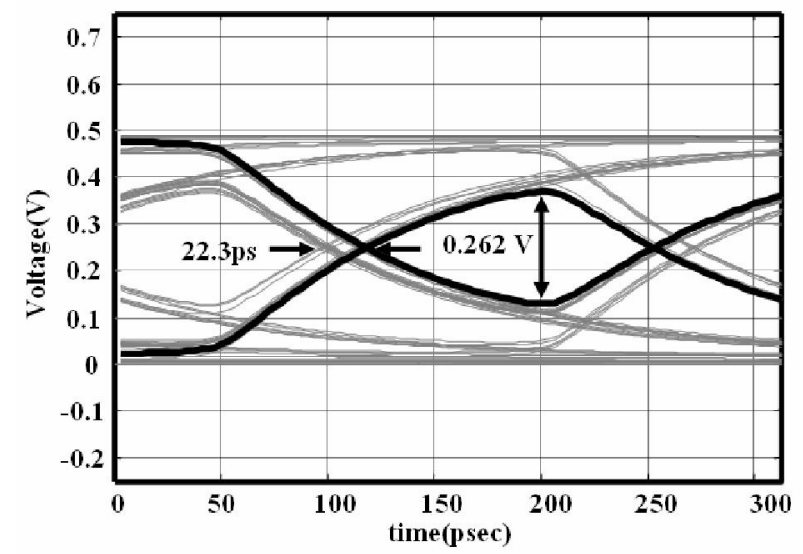

(a)

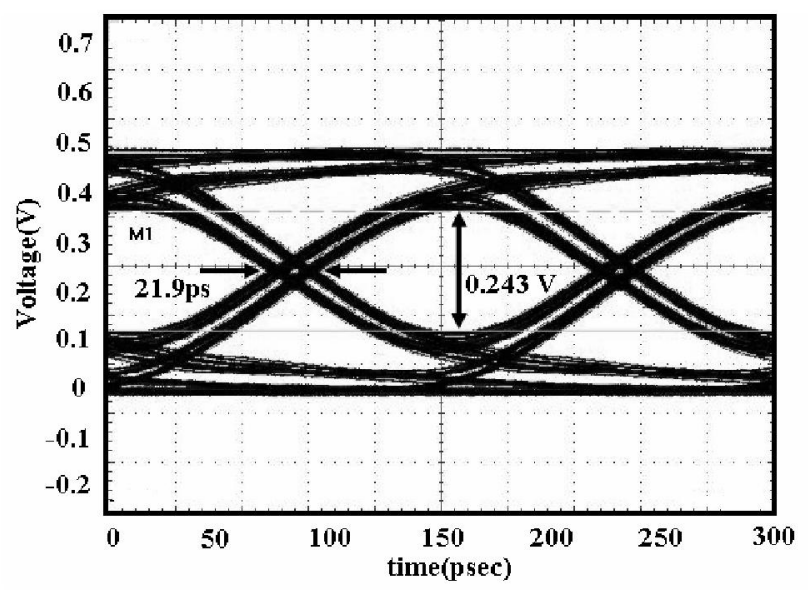

(b)

Figure 12 Comparison of (a) the estimation with (b) the measurement at the bit rate of $6.4 \mathrm{~Gb} / \mathrm{s}$

\section{Conclusions}

We introduced a simple and precise method to estimate data-dependent jitter in high-speed transmission lines with single pulse response from the frequency-dependent model of the channel. And the estimated data-dependent jitter and eye-height with the proposed method was verified by the timedomain jitter measurement performed in several conditions and found to be quite accurate.

The available bandwidth of the channel is broadened by numerical conversion of the domains using fast-fourier transform, thus the timing accuracy of the estimation is improved in high-speed data channel. Because of the simplicity of the method, the time required for calculation takes only a few seconds. And this will be useful for setting a timing budget in high-speed data channel.

\section{References}

1. Wavecrest Corp., "Jitter Fundamentals," application note,2005;http://www.wavecrest.com/technical/pdf/Jit ter_Fundamentals.pdf

2. Nelson Ou, T. Farahmard et al, "Jitter Models for Design and Test of Gbps-speed Serial Interconnects", IEEE Design \& Test Computers, 2004

3. James Buckwalter, Ali Hajimiri, "Predicting Datadependent Jitter", IEEE Trans. on Circuits and Systems, 2004

4. Eric Bogatin, "Signal Integrity", PTR Prentice Hall",2003, pp. 60

5. Eakhwan Song, Joungho Kim et al, "Data-dependent jitter estimation using singlepulse analysis method", Electronic Packaging Technology Conference, 2005

6. K.C.Gupta, Ramesh Garg et al, "Microstrip Lines and Slotlines", Artech House, 1996

7. Albert Sebban, K. C. Gupta, "A Planar-lumped Model for Coupled Microstrip Lines and Discontinuities", IEEE Trans. on Microwave Theory and Techniques, Vol. 40, No. 2, 1992 